\title{
Subtotal capsulectomy for idiopathic chondrolysis of the hip \\ A clinical, radiological and histological study
}

\author{
M Laubscher MBChB(UFS), Dip PEC, FC Orth(SA), MMed Ortho(UCT) \\ Consultant Orthopaedic Surgery \\ E Banderker MBChB, FC Rad Diag(SA) \\ Consultant Paediatric Radiology \\ K Pillay MBChB(UKZN), FC Path(SA), FRC Path(UK), MMed Anat Path(UCT) \\ Consultant Histopathology \\ M Held MD, FC Orth(SA), MMed Ortho(UCT) \\ Consultant Orthopaedic Surgery \\ S Dix-Peek MBChB, FC Orth(SA), MMed Ortho(UCT) \\ Consultant Paediatric Orthopaedic Surgery \\ EB Hoffman MBChB(US), FC Orth(SA) \\ Emeritus Associate Professor, Department of Orthopaedics, University of Cape Town
}

From Maitland Cottage Paediatric Orthopaedic Hospital and Red Cross War Memorial Children's Hospital, Cape Town, South Africa

\section{Corresponding author: \\ Dr Maritz Laubscher}

Email: maritzl@msn.com

\begin{abstract}
Background: Idiopathic chondrolysis (IC) of the hip is a rare, disabling condition affecting mainly adolescent females. Cartilage necrosis results in a stiff, painful hip which in the majority of cases have a poor outcome. The aetiology still remains unknown and the treatment is controversial and relatively unsuccessful. Subtotal capsulectomy with soft tissue releases has been reported to give good results.

Methods: Five female adolescents with IC of the hip were reviewed in order to shed light on the aetiology, delineate the role of magnetic resonance imaging (MRI) pre- and post-operatively and assess the outcome of a subtotal capsulectomy. The surgical technique was performed as originally described with concomitant releases of the deformities. Samples of synovium and cartilage were sent for histology and culture (including tuberculosis culture). Pre- and post-operatively the patients were evaluated regarding pain, deformity and range of motion (ROM). Follow-up radiographs and MRI scans were performed and compared to pre-operative imaging.

Results: Blood tests for auto-immune markers were negative in all cases. MRI pre-operatively showed erosions and mainly destruction of the superomedial acetabular cartilage. Histology of the synovium showed chronic, non-specific inflammation with a plasma cell infiltrate, suggesting an auto-immune cause. Histology of the cartilage confirmed cartilage necrosis. At follow-up (mean 11 months) four of the five patients were pain free and one patient reported an improvement in pain. There was an improvement in deformity and range of motion in four cases. Post-operative imaging (radiographs and MRI) at a mean of eight months, however, showed deterioration of the pathology with increase of the erosions, joint space narrowing and cartilage destruction.

Conclusions: We conclude that the aetiology is most likely an auto-immune process. MRI was helpful in delineating the cartilage pathology. A subtotal capsulectomy offers early post-operative clinical relief. Radiological deterioration however suggests that the surgery does not prevent deterioration of pathology.

Level of evidence: Level IV: Case series
\end{abstract}

Key words: idiopathic chondrolysis, hip, subtotal capsulectomy 


\section{Introduction}

Idiopathic chondrolysis of the hip is a very rare disorder that occurs mostly in adolescents with fewer than 130 cases documented. ${ }^{1}$ It is characterised by rapid cartilage necrosis of the hip joint, not associated with trauma, slipped upper femoral epiphysis (SUFE), infection or other demonstrable causes. ${ }^{2}$ Patients present with insidious onset of pain, a limp and a typical flexion, abduction and external rotation deformity of the hip joint.

It was first described in nine adolescents by Jones in 1971. The disease did not conform to other known conditions justifying the recognition of a distinct clinical entity. ${ }^{3}$ In 1982 Sparks and Dall from the same unit reported on the original series and a further nine patients. They confirmed the existence of the condition and reported a progressive downhill course with a poor outcome in $88 \%$ of cases despite different treatment strategies. ${ }^{4}$ Subsequently the condition was verified with reported cases from around the world..$^{5-15}$

Various treatment strategies have been advocated. Nonoperative treatment with non-steriodal anti-inflammatory drugs (NSAIDs), non-weight bearing and physiotherapy, including continuous passive motion (CPM), are used. ${ }^{16-18}$ In 1988 Roy and Crawford reported on a subtotal capsulectomy with soft tissue releases followed by aggressive rehabilitation..$^{19}$ Three cases were treated with good results. Subsequent studies reported indifferent results with the same technique. ${ }^{20}$

The purpose of our study was to try and shed light on the aetiology, to delineate the role of MRI and to assess the outcome of subtotal capsulectomy.

\section{Materials and methods}

Five adolescent female patients with a mean age of 11 years (range 10 to 12 years) were treated for IC from January 2011 to August 2012. The median duration of symptoms at presentation was 2 months (range 2-24 months). We performed a retrospective review of prospectively collected data on these patients. No patients were excluded from analysis.

Pre-operatively the patients were assessed clinically. Blood work-up included full blood count, erythrocyte sedimentation rate (ESR), rheumatoid factor, antinuclear factor and anti-neutrophil cytoplasmic antibodies. A chest radiograph and a Mantoux skin test were done. Pelvic radiograph, computed tomography (CT) and MRI scans of the hip were performed. Standardised MRI anatomical imaging planes using conventional joint imaging protocols as well as cartilage-specific imaging sequences were employed to distinguish the different zones of cartilage (physeal, epiphyseal and articular cartilage) in children. In addition to the routine sequences used for the evaluation of cartilage in adults (intermediate/proton density with fat suppression and T1-weighted spoiled gradient recalled echo with fat suppression), we also employed T2-weighted sequences that help differentiate the low signal epiphyseal cartilage from the higher signal physeal and articular cartilages. ${ }^{21}$

All patients underwent an extensive course of nonoperative management that included NSAIDs, physiotherapy and CPM. All five patients underwent subtotal capsulectomy with soft tissue releases. The surgical technique was as described by Roy and Crawford. ${ }^{19} \mathrm{~A} 1 \mathrm{~cm}$ strip of hip capsule was excised for an arc of $270^{\circ}$. The posterior $90^{\circ}$ was spared to preserve the blood supply to the femoral head. A psoas tenotomy at the pelvic brim and a release of the straight head of the rectus femoris muscle were done to release the flexion contracture of the hip. The tensor fascia lata was released to improve the abduction deformity. Synovial biopsies were sent for histology and culture (including TB culture) and a cartilage biopsy taken from the periphery of the femoral head was sent for histology.

Post-operatively the patients were initially treated in bilateral skin traction. After 7 to 10 days physiotherapy, including CPM, was initiated. Patients received NSAIDs for 6 weeks.

At a mean follow-up of 11 months (range 9-16 months) the patients were assessed for pain and ROM. Follow-up radiographs and MRI scans were done at a mean of 8 months to assess the disease progression.

\section{Results}

All the patients presented with pain and stiffness of the affected hip. Examination revealed an antalgic, Trendelenburg gait and a painful, stiff hip with a flexion, abduction and external rotation deformity in all cases (Table I). All blood tests including the auto-immune markers were normal. Chest radiographs were clear and Mantoux skin tests negative in all cases.

Initial pelvic radiographs revealed osteopaenia, concentric joint space narrowing (less than $3 \mathrm{~mm}$ ) and the deformity was visible in all cases (Figure 1). In one case subchondral erosions were visible on plain radiograph. Two cases fulfilled radiological criteria for protrusio bilaterally. CT confirmed osteopaenia and joint space narrowing in all cases, and subchondral erosions were seen in three cases (Figure 2). MRI scan at presentation was performed in four patients (it was not performed in one case due to resource restrictions). In all four cases where an initial MRI was performed joint space narrowing and marrow oedema was seen (Figure 3- $A$ ).

Table I: Pre-operative and latest follow-up deformity and ROM Mean value (range) Pre-operative Latest follow-up

\begin{tabular}{|l|c|c|}
\hline Deformity & & \\
\hline Fixed-flexion deformity & $31^{\circ}\left(20^{\circ}-45^{\circ}\right)$ & $26^{\circ}\left(20^{\circ}-30^{\circ}\right)$ \\
\hline Abduction deformity & $22^{\circ}\left(10^{\circ}-30^{\circ}\right)$ & $13^{\circ}\left(0^{\circ}-30^{\circ}\right)$ \\
\hline External rotation deformity & $20^{\circ}\left(10^{\circ}-30^{\circ}\right)$ & $8^{\circ}\left(0^{\circ}-30^{\circ}\right)$ \\
\hline Range of motion & & \\
\hline Flexion-extension arc & $25^{\circ}\left(0^{\circ}-60^{\circ}\right)$ & $40^{\circ}\left(30^{\circ}-60^{\circ}\right)$ \\
\hline
\end{tabular}




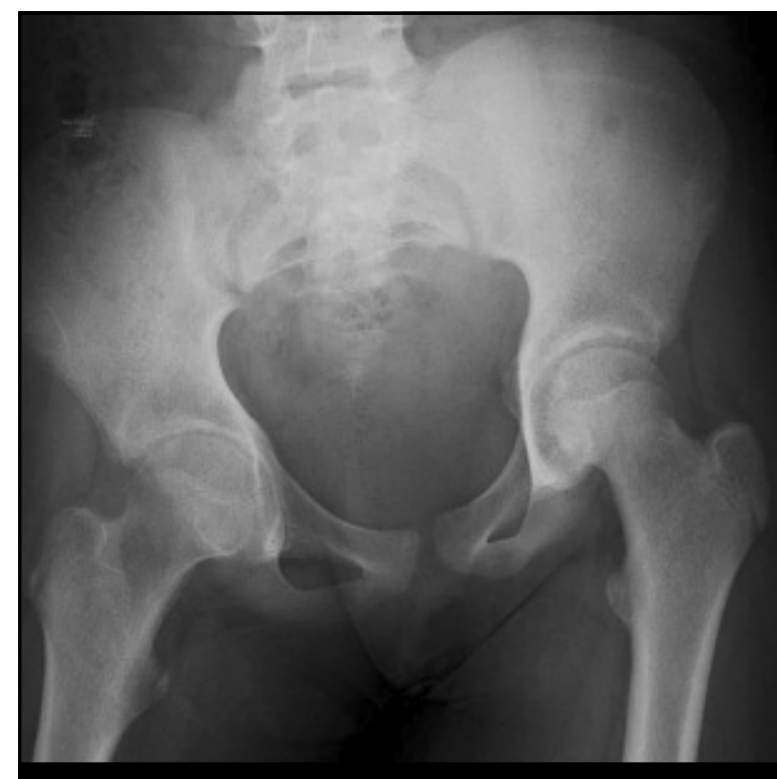

Figure 1. A-P radiograph of the pelvis of an 11-year-old girl with IC of the right hip showing typical findings of osteopaenia, joint space narrowing, closure of the femoral capital physis and flexion, abduction and external rotation deformities

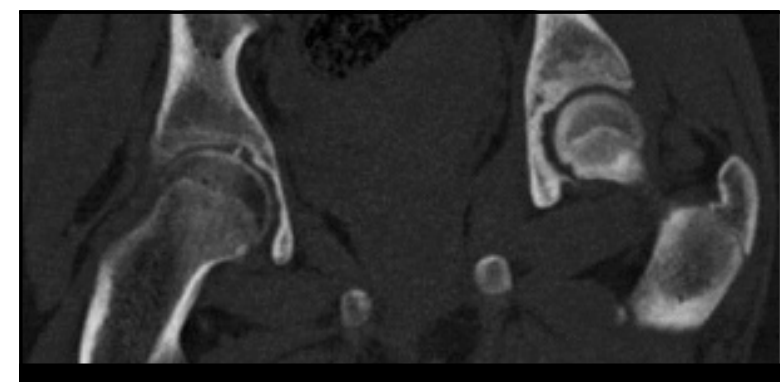

Figure 2. Coronal CT scan of the same patient confirming joint space narrowing and also showing a subchondral erosion on the acetabular side. Also note the demineralisation as compared to the normal left hip.

Subchondral erosions were seen in three out of four cases (Figure 3-B). The cartilage loss and erosions were mostly acetabular and mainly in the superomedial weight bearing area of the acetabulum (Figure 3-D). Slight synovial enhancement was seen in one case.

Non-operative treatment failed in all cases. Histological examination of the synovium showed signs of mild, nonspecific chronic inflammation with a perivascular infiltrate of lymphocytes and plasma cells in all cases. There was no evidence of granulomatous inflammation (Figure 4-A). Macroscopically, the cartilage appeared lustreless. Microscopically, there was an outer layer of fibroblastic tissue with an adjacent layer of degenerate chondrocytes. There were viable chondrocytes at the base (Figure 4-B). Microbiological culture was negative in all cases, including extended TB culture.
At the last follow-up all patients subjectively felt that their pain had improved. Four patients felt that their hips were pain-free. The gait improved in four out of the five cases. The deformity was improved in four cases. In one case the patient's hip was still painful and there was no improvement in the deformity (Table I).

Post-operative radiographs showed deterioration of the joint space narrowing in four of the five cases. On follow-up MRI subchondral erosions were now visible in all five hips and cartilage destruction was worse compared to the pre-operative MRI in all four cases with pre-operative MRI.

\section{Discussion}

Idiopathic chondrolysis occurs mostly unilaterally, although bilateral cases have been described. ${ }^{22,23}$ It affects adolescents with a predominance among girls, but male cases and cases presenting shortly after skeletal maturity have been described. ${ }^{24}$ Initially it was thought to occur in individuals of African descent only, but subsequently cases have been documented from different races across the world. ${ }^{2,24}$ Literature is limited to case studies and small case series.

The presence of stiffness accompanied by radiographic osteopaenia and concentric reduction of the joint space to less than $3 \mathrm{~mm}$ can be considered a diagnostic criterion for IC. ${ }^{1,2}$ In our setting the main differential diagnosis is atrophic-type tuberculosis of the hip. ${ }^{25,26}$

\section{Aetiology}

The aetiology remains unknown. Theories include an auto-immune process in genetically susceptible individuals ${ }^{5,27}$ and mechanical theories. The most widely accepted theory is that the articular cartilage destruction is due to an auto-immune response in genetically susceptible individuals. ${ }^{1,228}$ Supporters of this theory have shown inflammatory cell infiltrates and immunoglobulin deposits in the synovium of the hip joints. ${ }^{5,27,30,31}$ Some authors have shown serological abnormalities. ${ }^{2}$ In our series auto-immune markers were all negative. A slightly elevated ESR of less than $30 \mathrm{~mm} / \mathrm{h}$ has been reported. ${ }^{32}$ The synovial inflammatory infiltrate, especially plasma cells, supports the theory of an auto-immune disease. This suggests that the powerful anti-inflammatory properties of the tumour necrosis factor-blockers might be useful. There is a single case study of successful treatment with etanercept. $^{33}$

Mechanical theories for IC are a decrease in synovial nutrition, $^{29,34}$ a mechanical insult with release of chondrolytic enzymes ${ }^{2}$ or an increase in intracapsular pressure which is supposedly released by subtotal capsulectomy. ${ }^{19}$ In SUFE the mechanical theory is supported by the fact that persistent pin or screw penetration in the antero-superior weight-bearing quadrant of the femoral head resulted in chondrolysis. ${ }^{35,36}$ 


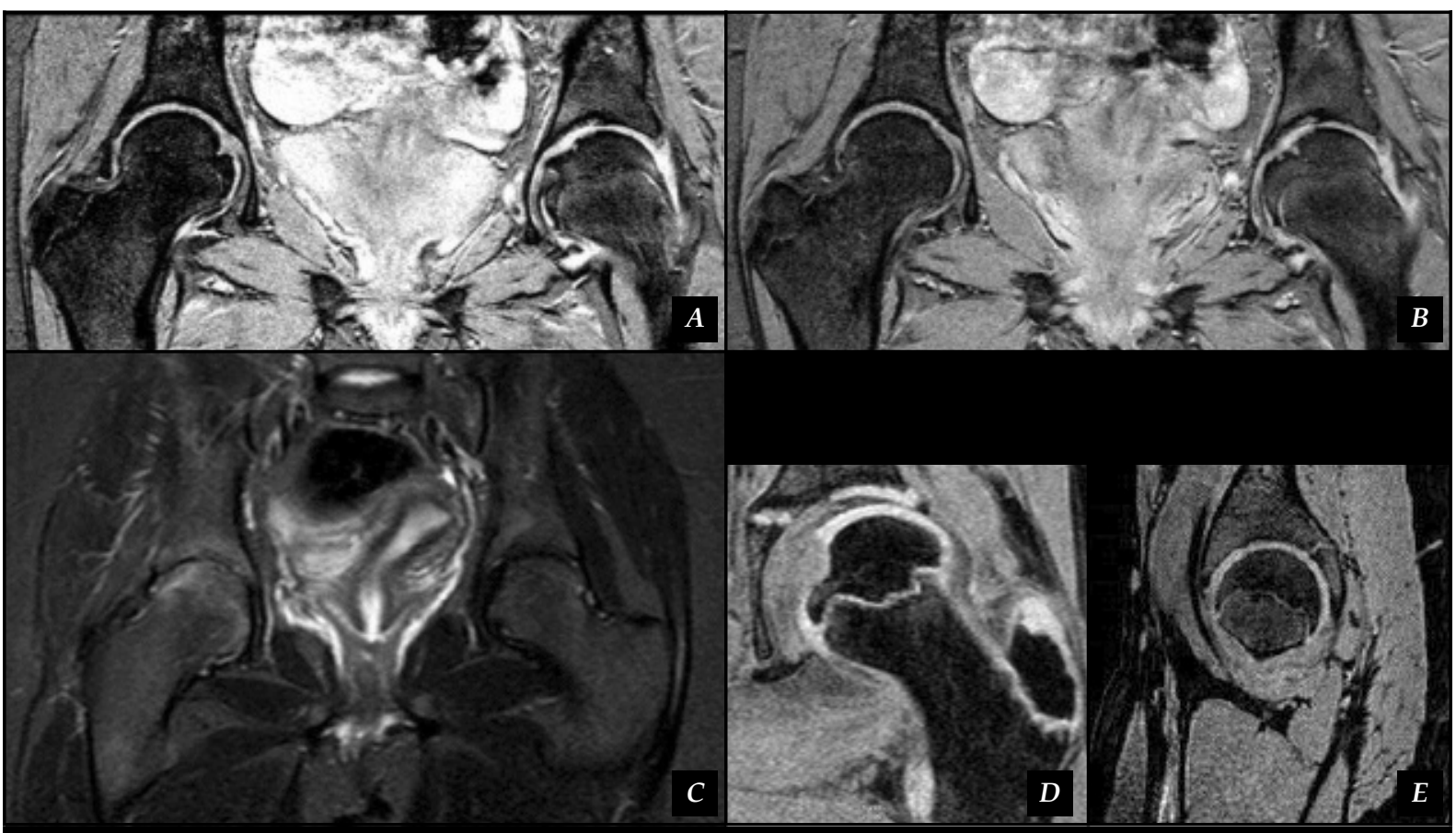

Figure 3. Representative images from our series to demonstrate typical MRI features:

A) Normal right hip: Coronal T2-weighted (T2W) Fast Field Echo (FFE) sequence showing normal high signal acetabular and femoral head cartilage

B) Abnormal left hip: Coronal T2W FFE sequence with fat suppression showing joint space narrowing, loss of both acetabular and femoral head cartilage (most evident along the superior joint compartment) and small femoral head subchondral bone cysts

C) Marrow oedema: Coronal Short T1 Inversion Recovery (STIR) sequence showing contiguous right femoral head and acetabular high signal marrow oedema

D) Normal cartilage: Coronal T1W 3D THRIVE (TR 8.1; TE 3.7) sequence showing normal acetabular and femoral cartilage

E) Acetabular cartilage loss: Sagittal T1W 3D THRIVE (TR 8.1; TE 3.7) sequence showing acetabular cartilage loss

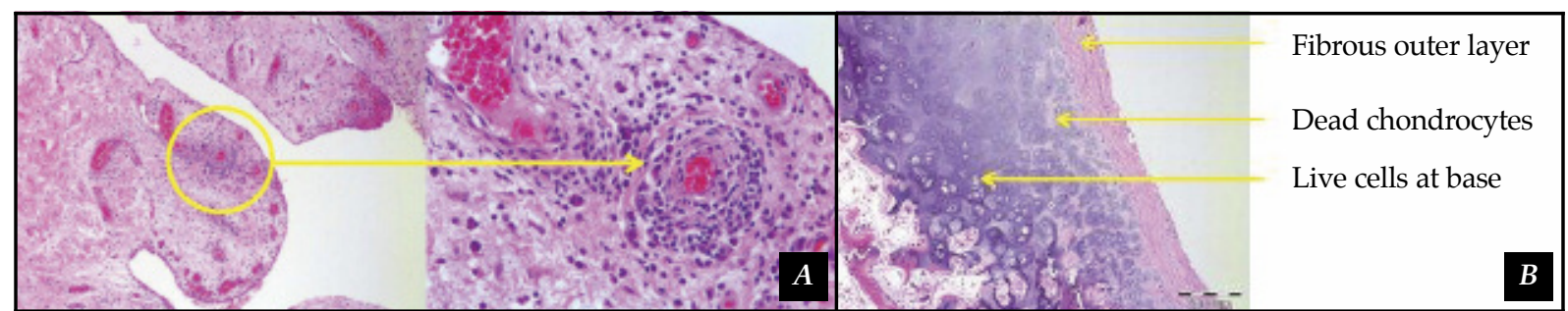

Figure 4: Histological findings (haematoxylin and eosin stains were utilised)

A) Synovial biopsy showing a perivascular infiltrate of lymphocytes and plasma cells

B) Cartilage biopsy showing an outer layer of fibroblastic tissue with an adjacent layer of degenerate chondrocytes and viable chondrocytes at the base 
Hips with transient penetration or penetration of the postero-inferior quadrant did not develop chondrolysis. Removal of the penetrating metal ware had a $75 \%$ chance of recovery. Those patients however, who had chondrolysis at presentation before surgery, were all female of African descent with chronic moderate or severe slips, suggesting a genetic and mechanical cause. The results of these hips are poor in the majority of cases, ${ }^{36}$ which is similar to that of IC reported from the same unit. ${ }^{4}$

Some authors suggest IC to be a form of cartilaginous dysplasia. ${ }^{37}$

Ippolito et al. in an ultrastructural study of the articular cartilage in IC found no resemblance to the chronic degenerative changes seen in osteoarthritis. They concluded IC to be a pathological process which, unlike osteoarthritis, runs its course very quickly. ${ }^{30}$

\section{Imaging}

Plain radiography is the initial investigation of choice and in the appropriate clinical setting, no further radiological investigation is necessary. Radiographs are frequently normal early on in the disease process. ${ }^{1}$ Subsequent findings reveal joint space narrowing, juxta-articular osteopaenia and small subchondral irregularities together with remodelling of the femoral neck and epiphysis. ${ }^{38}$ Premature closure of the femoral capital physis ${ }^{9}$ and protrusio acetabuli ${ }^{39}$ is also documented. Late manifestations of the disease reflect the secondary degenerative changes of osteoarthritis. ${ }^{4}$

Our investigation demonstrated the typical plain radiographic findings of joint space narrowing, juxtaarticular osteopenia and joint deformity. In addition, two cases revealed bilateral protrusio acetabuli according to radiographic criteria. Some authors have implicated the same disease process in IC and primary acetabular protrusio despite their distinct natures..$^{39}$ Only one case in our series demonstrated subchondral erosions on the initial radiograph.

$\mathrm{CT}$, though superior in depicting anatomical detail and in its sensitivity for detecting subchondral bone erosions (three out of five cases at presentation), its benefit over and above plain radiography is negated by the radiation burden, particularly in the vulnerable paediatric setting. Hence this has subsequently been excluded from our diagnostic workup.

We found MRI useful in aiding diagnosis in difficult cases as well as assessing progression of the disease. MRI findings are divided into early and late features. Early features included focal cartilage loss, bone marrow oedema, a small joint effusion without significant synovial enhancement, muscle wasting and signs of bone remodelling. ${ }^{38,40,41}$ Late features were widespread cartilage loss and bone erosions..$^{38}$

Our case series demonstrated similar features with the notable exception of early articular cartilage destruction affecting the acetabular cartilage (superomedial weightbearing areas) more than the femoral head cartilage as suggested by earlier reports. ${ }^{38,40}$ Synovial enhancement was documented in one of four of the initial MRI studies in our series. Slight synovial enhancement has been described in the early phase of IC. ${ }^{40}$ Significant synovial enhancement is however more suggestive of juvenile idiopathic arthritis (JIA) or chronic infections including TB. MRI is therefore useful in both the diagnostic workup and for the follow-up of disease progression in IC and is routinely employed in suspected cases in our practice.

The only realistic differential diagnosis to be entertained in the local clinical scenario is that of atrophic-type tuberculosis of the hip. A study by Reardon et al. compared radiographs of eight patients with IC and nine with atrophic tuberculosis. Plain radiographs of the TB hips showed peri-articular erosions which were absent in IC. ${ }^{26}$ In the current study three hips had subchondral erosions at presentation on CT and MRI, mainly superomedially in the acetabulum. Monoarthritis of the hip in JIA is invoked as a likely mimic in the literature, ${ }^{1}$ but has never been documented at our institution.

\section{Outcome}

The natural history of idiopathic chondrolysis is unpredictable. A variety of outcomes from spontaneous resolution to painful ankylosis has been described. ${ }^{1,3,4}$ There appears to be two stages in the disease with an initial acute stage with onset of symptoms caused by an inflammatory response. This is followed by a chronic phase with an unpredictable course. $^{1,2}$

Several authors describe spontaneous resolution of the condition over time., ${ }^{9,10,42}$ This is not our experience, with none of our patients responding to prolonged non-operative management.

An interesting finding was that the ROM improved under general anaesthetic prior to surgery in all cases. This confirms that muscle spasm contributes to the deformity as shown by previous authors. ${ }^{3}$

Roy and Crawford in 1988 described a subtotal capsulectomy with soft tissue releases and aggressive rehabilitation in three cases. ${ }^{19}$ Although intracapsular pressure was never measured they felt it relieved increased intracapsular pressure. They described good results with improvement in pain and range of motion in all cases, which was reproduced by some authors. ${ }^{13}$ They also reported restoration of the joint space on radiographs..$^{19}$ Other authors however reported clinical and radiological progression of the disease following similar surgical procedures ${ }^{39,40,43}$ and early clinical improvement with radiological deterioration and no long-term benefit. ${ }^{20}$ In all our cases the pain was improved following surgery, with four out of five cases pain-free. The deformity was also improved in four cases, with a slight improvement of the ROM of the hip. In all five cases though there was radiological progression. On plain radiograph there was deterioration of the joint space narrowing and more subchondral erosions were visible. 
Follow-up MRI revealed more severe cartilage destruction in all cases. From these findings we conclude that although the subtotal capsulectomy offers some relief of pain and deformity, it does not alter the progression of the disease. It does however allow the hip to stiffen up in a more favourable position.

Other treatment modalities have been described. Aldegheri et al. reported clinical improvement despite radiological deterioration in cases of articulated distraction for chondrolysis secondary to SUFE. ${ }^{44}$ Articulated distraction has also been described for the adolescent arthritic hip due to other causes with encouraging results. ${ }^{45}$ Our experience with this technique in chondrolysis in SUFE was disappointing. Salter et al. in 1980 demonstrated the effect of CPM on healing of full thickness defects in articular cartilage. ${ }^{46}$ In theory it should be possible to address acetabular cartilage loss with chondrogenesis through metaplasia by drilling subchondral bone and CPM. The viable chondrocytes shown in the basal cartilage at histology may benefit from this treatment. The use of biological agents like etanercept is also a possibility as discussed earlier. ${ }^{33}$ More research is needed on these novel treatments.

\section{Limitations}

The main limitation of our study is the retrospective nature as well as the lack of a clinical scoring system to evaluate outcome. We also had a small series, but due to the extreme rarity of the condition most studies on the topic are limited to small case series.

\section{Conclusions}

We conclude that the aetiology is most likely an autoimmune process. MRI was helpful in delineating the cartilage pathology, which was mainly on the acetabular side. A subtotal capsulectomy offers early post-operative clinical relief. Radiological deterioration suggests that the surgery does not prevent progression of the pathology.

\section{Conflict of interest statement}

None of the authors received funding or reported a conflict of interest regarding this study. This study is not based on a previous communication.

\section{References}

1. Segaren N, Abdul-Jabar HB, Segaren N, Hashemi-Nejad A. Idiopathic chondrolysis of the hip: presentation, natural history and treatment options. J Pediatr Orthop B. 2014;23(2):112-16.

2. Dobbs MB, Morcuende JA. Other conditions of the hip. In: Morrissy RT, Weinstein SL, eds. LovellEWinter's Pediatric Orthopaedics. 6th ed. Philadelphia: Lippincot Williams\&Wilkens; 2006:1147-52.

3. Jones BS. Adolescent chondrolysis of the hip joint. South African Med J. 1971;(February):196-202.

4. Sparks LT, Dall G. Idiopathic chondrolysis of the hip joint in adolescents. South African Med J. 1982;61(June):883-86.
5. Moule NJ, Golding JSR. Idiopathic chondrolysis of the hip. Clin Radiol. 1974;25(2):247-51.

6. Wenger DR, Mickelson MR, Ponseti I V. Idiopathic chondrolysis of the hip. Report of two cases. J Bone Joint Surg Am. 1975;57(2):268-71.

7. Duncan JW. The Bizarre Stiff Hip. JAMA. 1975;231(4):382.

8. Smith EJ, Ninin DT, Keays AC. Idiopathic chondrolysis of the hip. A case report. South African Med J. 1983;63(3):8890.

9. Bleck EE. Idiopathic chondrolysis of the hip. J Bone Joint Surg Am. 1983;65(9):1266-75.

10. Daluga DJ, Millar EA. Idiopathic chondrolysis of the hip. J Pediatr Orthop. 1989;9(4):405-11.

11. Donnan L, Einoder B. Idiopathic chondrolysis of the hip. ANZ J Surg. 1996;66(8):569-71.

12. Kozlowski K, Scougall J. Idiopathic chondrolysis diagnostic difficulties. Pediatr Radiol. 1984;14(5):314-17.

13. Nutthapong Wongwiwat M. Idiopathic chondrolysis of the hip: A case report and review of the literature. Thai $J$ Orthop Surg. 2006;31(2):94-99.

14. Sivanantham M, Kutty MK. Idiopathic chondrolysis of the hip: case report with a review of the literature. ANZ J Surg. 1977;47(2):229-31.

15. Van der Hoeven H, Keessen W, Kuis W. Idiopathic chondrolysis of the hip: A distinct clinical entity? Acta Orthop Scand. 1989;60(6):661-63.

16. Hughes AW. Idiopathic chondrolysis of the hip: a case report and review of the literature. Ann Rheum Dis. 1985;44(4):268-72.

17. Bilski P, Snela S. Difficulties in treating chondrolysis and avascular necrosis of the hip in adolescent patients. Ortop Traumatol Rehabil. 2006;8(1):34-40.

18. François J, Mulier M. Idiopathic chondrolysis of the hip: A case report. Acta Orthop Belg. 2007;73(5):653-57.

19. Roy DR, Crawford AH. Idiopathic chondrolysis of the hip: management by subtotal capsulectomy and aggressive rehabilitation. J Pediatr Orthop. 1988;8:203-207.

20. Del Gouz Garcia A, Fernandez PL, Gonzalez MPC, Garcia AC, Gonzalez LR, Jimenez JP. Idiopathic chondrolysis of the hip: Long-term evolution. I Pediatr Orthop. 1999;19(4):449-54.

21. Jaramillo D. Cartilage imaging. Pediatr Radiol. 2008;38 Suppl 2:256-258.

22. Rachinsky I, Boguslavsky L, Cohen E, Hertzanu Y, Lantsberg S. Bilateral idiopathic chondrolysis of the hip. Clin Nucl Med. 2000;25(12):1007-1009.

23. Rowe LJ, Ho EK. Idiopathic chondrolysis of the hip. Skeletal Radiol. 1996;25(2):178-82.

24. Ippolito E, Ricciardi-Pollini PT. Chondrolysis of the hip (idiopathic and secondary forms). Ital J Orthop Traumatol. 1981;7(3):335-44.

25. Campbell JAB, Hoffman EB. Tuberculosis of the hip in children. J Bone Joint Surg Br. 1995;77(2):319-26.

26. Reardon TC, Holm H, Solomon R, Sparks LT, Hoffmann EB. Idiopathic chondrolysis versus atrophic tuberculosis of the hip in children: the problem of differential diagnosis. J Bone Jt Surgery, Br Vol. 2003;85-B(SUPP II):148.

27. Eisenstein A, Rothschild S. Biochemical abnormalities in patients with slipped capital femoral epiphysis and chondrolysis. J Bone Joint Surg Am. 1976;58(4):459-67. 
28. Herman JH, Herzig EB, Crissman JD, Dennis M V, Hess E V. Idiopathic chondrolysis-an immunopathologic study. J Rheumatol. 1980;7(5):694-705.

29. Waldenstrom H. On necrosis of the joint by epiphysiolysis capitis femoris. Acta Chir Scand. 1930;67:936-46.

30. Ippolito E, Bellocci M, Santori FS, Ghera S. Idiopathic chondrolysis of the hip: an ultrastructural study of the articular cartilage of the femoral head. Orthopedics. 1986;9(10):1383-87.

31. Morrissy RT, Kalderon AE, Gerdes MH. Synovial immunofluorescence in patients with slipped capital femoral epiphysis. J Pediatr Orthop. 1981;1(1):55-60.

32. Duncan J, Nasca R, Schrantz J. Idiopathic chondrolysis of the hip. J Bone Joint Surg Am. 1979;61(7):1024-28.

33. Appleyard D V, Schiller JR, Eberson CP, Ehrlich MG Idiopathic chondrolysis treated with etanercept. Orthopedics. 2009;32(3):214.

34. Cruess RL. The pathology of acute necrosis of cartilage in slipping of the capital femoral epiphysis. A report of two cases with pathological sections. J Bone Joint Surg Am. 1963;45(5):1013-24.

35. Vrettos BC, Hoffman EB. Chondrolysis in slipped upper femoral epiphysis. Long-term study of the aetiology and natural history. J Bone Joint Surg Br. 1993;75(6):956-961.

36. Nortje MB, Dix-Peek S, Vrettos BC, Hoffman EB. Avascular necrosis and chondrolysis in slipped upper femoral epiphysis: A comparative study between multiple pin fixation with or without osteotomy and single screw fixation. South African Orthop J. 2009;(Summer):30-37.

37. Stanescu R, Stanescu V, Maroteaux P, Peyron J. Constitutional articular cartilage dysplasia with accumulation of complex lipids in chondrocytes and precocious arthrosis. Arthritis Rheum. 1981;24(7):965-68.
38. Johnson K, Haigh SF, Ehtisham S, Ryder C, GardnerMedwin J. Childhood idiopathic chondrolysis of the hip: MRI features. Pediatr Radiol. 2003;33(3):194-99.

39. Sherlock DA. Acute idiopathic chondrolysis and primary acetabular protrusio may be the same disease. J Bone Joint Surg Br. 1995;77(2):392-95.

40. Laor T, Crawford AH. Idiopathic chondrolysis of the hip in children: early MRI findings. Am J Roentgenol. 2009;192(2):526-31.

41. Mounach A, Nouijai A, Ghozlani I, et al. Idiopathic chondrolysis of the hip. Joint, bone, spine. 2007;74(6):65658.

42. Lejman T, Sulko J. Idiopathic chondrolysis of the hip. Chir Narzadow Ruchu Ortop Pol. 2005;70(2):97-100.

43. Korula RJ, Jebaraj I, David KS. Idiopathic chondrolysis of the hip: medium- to long-term results. ANZ J Surg. 2005;75(9):750-53.

44. Aldegheri R, Trivella G, Saleh M. Articulated distraction of the hip conservative surgery for arthritis in young patients. Clin Orthop Relat Res. 1994;301:94-101.

45. Thacker MM, Feldman DS, Madan SS, Straight JJ, Scher DM. Hinged distraction of the adolescent arthritic hip. $J$ Pediatr Orthop. 2005;25(2):178-82.

46. Salter R, Simmonds D, Malcolm B, Rumble E, MacMichael $\mathrm{D}$, Clements $\mathrm{N}$. The biological effect of continuous passive motion on the healing of full-thickness defects in articular cartilage. An experimental investigation in the rabbit. $J$ Bone Joint Surg Am. 1980;62(8):1232-51.

This article is also available online on the SAOA website (www.saoa.org.za) and the SciELO website (www.scielo.org.za). Follow the directions on the Contents page of this journal to access it. 